\title{
Prolonged Ictal Aphasia Presenting as Clinical- Diffusion Mismatch in a Patient with Acute Ischemic Stroke
}

\author{
Joo Yea Jin, Yeon-Jung Kim, Sun U. Kwon \\ Department of Neurology, Asan Medical Center, Seoul, Korea
}

\section{Dear Sir:}

The presence of clinical-diffusion mismatch (CDM) in patients with acute ischemic stroke may represent the ischemic penumbra, which requires emergency reperfusion therapy to improve stroke outcomes. We report here our experience of treating a patient with acute ischemic stroke and CDM. Specifically, the patient had a small infarct in the left temporal cortex and presented with sensory aphasia, but did not require reperfusion therapy. Magnetic resonance angiography and perfusion imaging findings were normal. Further investigation revealed that the aphasia was associated with ictal symptoms. Ictal aphasia is a considerable cause of non-oligemic CDM, perfusion imaging and angiographic studies may help discriminate true ischemic penumbra from non-oligemic CDM.

A 44-year-old, right-handed male patient presented with sudden onset language disturbance and headache for 24 hours. Approximately 30 years prior, the patient had undergone surgery to removal a right cerebellar tumor, which resulted in a history of mild dysarthria, hearing difficulties, right-sided ataxia, leftsided facial palsy, and right exotropia. There was no history of tumor recurrence or seizure. Neurologic examination revealed Wernicke's aphasia in addition to the preexisting neurological deficits, with an initial score on the National Institutes of Health Stroke Scale of seven. Diffusion-weighted magnetic resonance imaging of the brain revealed a small, focal, and high-signal intensity (volume $1.3 \mathrm{~mL}$ ) with the apparent diffusion coefficient restriction in the left temporal lobe, compatible with acute infarction (Figure 1). Because there were no lesions that could explain the patient's aphasia, we diagnosed the condition to be significant neurological deficit with $\mathrm{CDM}$ and considered emergency revascularization. However, the revascularization procedure was not performed because we did not note any abnormal findings on magnetic resonance angiography or perfusion imaging.

The results of the Korean version of the Western Aphasia Battery indicated severe Wernicke's aphasia (10/20 fluency, 1.9/10 auditory comprehension, $0.4 / 10$ repetition, $0.5 / 10$ naming, and 45.6/100 aphasia quotient). There were no abnormal laboratory results except a mild elevation in erythrocyte sedimentation rate $(23 \mathrm{~mm} / \mathrm{h})$. The electrocardiogram showed a normal sinus rhythm, and chest $\mathrm{x}$-ray showed no active lesion in either lung fields. During hospitalization, the severity of aphasia and mild confusion fluctuated. Electroencephalography demonstrated intermittent theta slowing in the left temporal areas (Figure 1). Fluorodeoxyglucose positron emission tomography (FDGPET) showed hypermetabolism in the left temporal cortex (Figure 1). The aphasia was due to ictal and postictal symptoms following stroke rather than hypoperfusion-related symptoms. The patient was administered $250 \mathrm{mg} /$ day phenytoin on the fourth day of admission. Symptoms remained on the following day, albeit attenuated; therefore, $1,000 \mathrm{mg} /$ day levetiracetam was added to the treatment regimen. Symptoms resolved on day 8 of admission; however, the patient complained of dizziness and depressive mood. We therefore changed his medication at discharge to include $600 \mathrm{mg} /$ day valproic acid, which was maintained for eight months. The patient has been seizurefree for one year (Figure 2), and the temporal hypermetabolism noted on FDG-PET has improved.

The predominant cause of sudden onset, prolonged aphasia, 


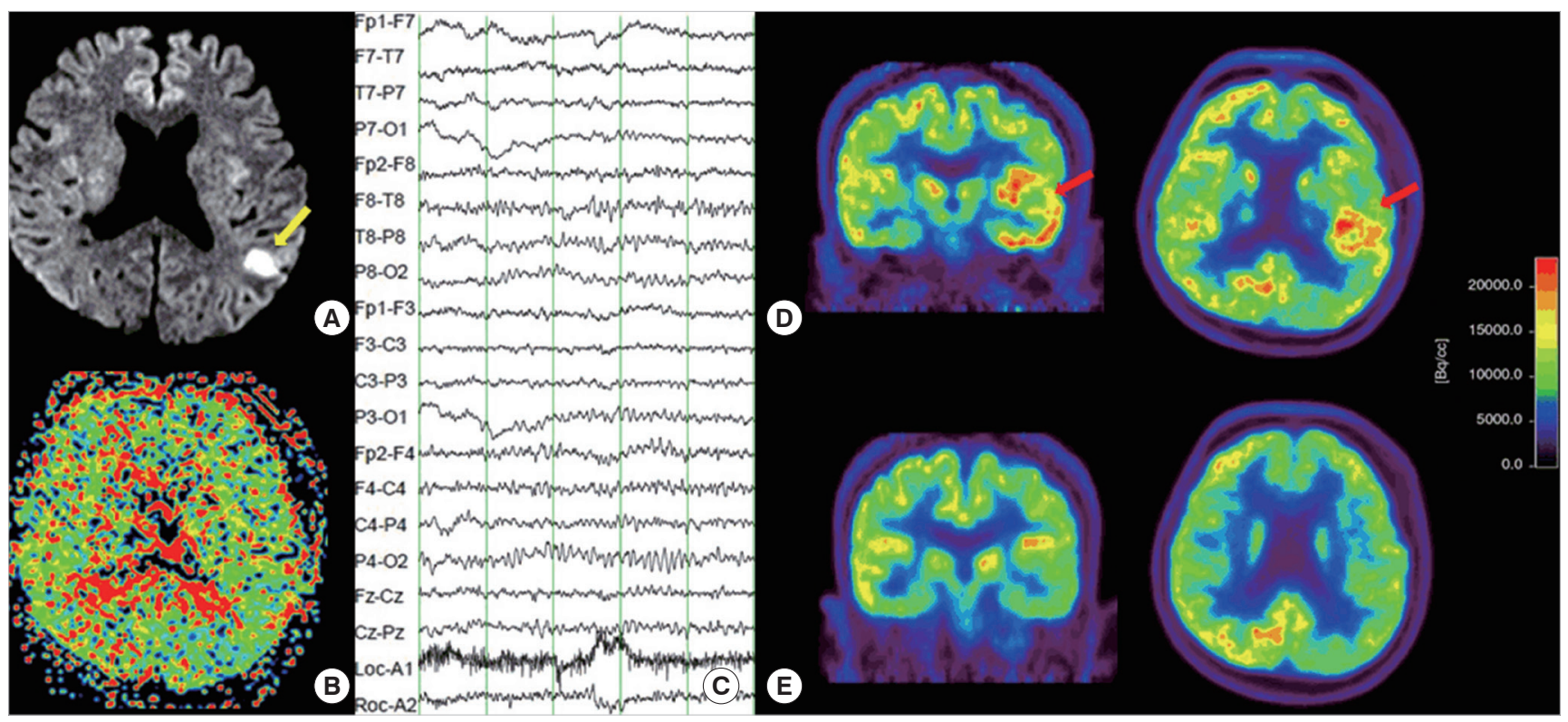

Figure 1. Representative images from magnetic resonance imaging and Fluorodeoxyglucose positron emission tomography (FDG-PET) of the brain. (A) Diffusionweighted image showing a small cortical infarct (yellow arrow) in the territory of the left middle cerebral artery. (B) Perfusion image showing no obvious abnormalities. (C) Electroencephalography showing intermittent theta slowing in the left temporal cortex. (D) Images of FDG-PET, which was performed at the onset of symptoms, showing hypermetabolism (red arrow) in the left temporal cortex (coronal and axial view). (E) Images of the regions shown in D obtained during follow-up FDGPET, which was performed when the symptoms resolved; they reveal normalization of left temporal hypermetabolism (coronal and axial view).

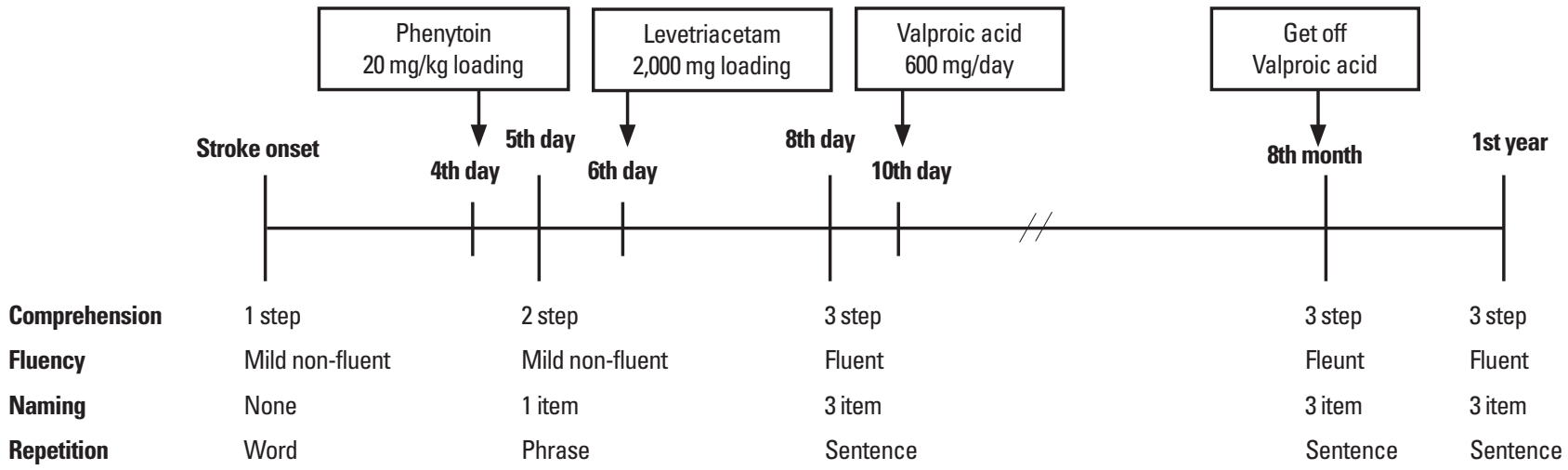

Figure 2. Hospital course of the patient showing the symptoms and prescribed medications.

is stroke affecting the language network. In the present case, the patient developed sudden onset Wernicke's aphasia, with a small cortical high-signal intensity in the territory of the left middle cerebral artery on magnetic resonance imaging. Because the lesion did not explain the patient's symptoms, the aphasia was initially considered a symptom resulting from hypoperfusion in the territory of the left middle cerebral artery, and was therefore considered CDM. However, the cause of aphasia was subsequently thought to be an ictal and postictal symptom caused by acute ischemic stroke, due to the following reasons: First, there were no steno-occlusive lesions on magnetic resonance angiography or perfusion defects, even though the symptoms lasted more than two days. Second, the small cortical lesion itself could not explain the intermittent slowing in the left temporal area found on electroencephalography. Third, FDG-PET performed when the aphasic symptoms presented, showed hypermetabolism in the left temporal lobe. Fourth, aphasic symptoms and hypermetabolism on FDG-PET disappeared after the administration of antiepileptic drugs. Due to its low prevalence, the diagnosis of ictal aphasia is difficult, and only $56 \%$ of patients with aphasic seizures demonstrate electrographic ictal discharge on the first routine surface electroencephalography. ${ }^{1}$ FDG-PET imaging was useful for the diagnosis of aphasic nonconvulsive status epilepticus, although the electroencephalography findings were inconclusive. ${ }^{2}$

According to previous studies, CDM is associated with per- 
fusion-diffusion mismatch, infarction expansion, and early neurological deterioration. ${ }^{3,4}$ It is therefore important to consider CDM when determining acute stroke management, such as thrombolysis or endovascular treatment. ${ }^{3,4}$ However, the falsepositive diagnosis of ischemic stroke, labeled "stroke mimics", ranges between $1.3-25 \%$, and seizures (38\%) are the most common cause of stroke mimics. ${ }^{5,6}$ Moreover, acute ischemic stroke is important in the etiology of symptomatic seizures.

Nonconvulsive seizures can be confused with CDM, as seen in the case reported here. Initially, revascularization was considered as a treatment for CDM. However, CDM can also be due to stroke mimics, indicating that clinicians should consider the possibility of post-stroke seizures. Perfusion imaging, magnetic resonance angiography and FDG-PET can provide useful information for differentiating non-oligemic $\mathrm{CDM}$ from true ischemic penumbra.

\section{References}

1. Ericson EJ, Gerard EE, Macken MP, Schuele SU. Aphasic status epilepticus: electroclinical correlation. Epilepsia 2011;52: 1452-1458.

2. Dong C, Sriram S, Delbeke D, Al-Kaylani M, Arain AM, Singh $\mathrm{P}$, et al. Aphasic or amnesic status epilepticus detected on PET but not EEG. Epilepsia 2009;50:251-255.

3. Davalos A, Blanco M, Pedraza S, Leira R, Castellanos M, Pumar
JM, et al. The clinical-DWI mismatch: a new diagnostic approach to the brain tissue at risk of infarction. Neurology 2004;62: 2187-2192.

4. Prosser J, Butcher K, Allport L, Parsons M, MacGregor L, Desmond $\mathrm{P}$, et al. Clinical-diffusion mismatch predicts the putative penumbra with high specificity. Stroke 2005;36:1700-1704.

5. Winkler DT, Fluri F, Fuhr P, Wetzel SG, Lyrer PA, Ruegg S, et al. Thrombolysis in stroke mimics: frequency, clinical characteristics, and outcome. Stroke 2009; 40:1522-1525.

6. Chernyshev OY, Martin-Schild S, Albright KC, Barreto A, Misra $\mathrm{V}$, Acosta I, et al. Safety of tPA in stroke mimics and neuroimaging-negative cerebral ischemia. Neurology 2010;74:13401345 .

Correspondence: Sun U. Kwon

Department of Neurology, Asan Medical Center, 88 Olympic-ro 43-gil, Songpa-gu, Seoul 183-736, Korea

Tel: +82-2-3010-3960

Fax: +82-2-474-4691

E-mail: sukwon@amc.seoul.kr

Received: January 28, 2014

Revised: April 7, 2014

Accepted: April 14, 2014

This study was supported by a grant from the Korea Healthcare Technology REtD Project, Ministry of Health and Welfare Republic of Korea (HI10C2020).

The authors have no financial conflicts of interest. 